\title{
De la limitation des psychothérapies
}

Bernard Scherler

Correspondance:

Dr Bernard Scherler

Spécialiste FMH en psychiatrie-

psychothérapie, Spécialiste FMH

en psychiatrie-psychothérapie

d'enfants et d'adolescents

Rue St-Maurice 12

CH-2000 Neuchâtel

Tél. 0327253823

scherlerb@bluewin.ch
Il apparaît que, par inadvertance ou par négligence coupable, je n'ai pas accordé aux numéros du BMS parus depuis début juillet toute l'attention qu'ils méritaient.

En particulier, j'ai manqué les réactions des mes confrères psychiatres et autres spécialistes à la décision du DFI de limiter la prise en charge des psychothérapies. J'ai complètement passé à côté de la réflexion de ce collègue qui s'inquiète de devoir guérir ses patients schizophrènes en 40 séances sous peine de se voir refuser une prolongation du traitement. De ce confrère gynécologue qui voit pointer à l'horizon des formulaires à remplir dès la $6^{\mathrm{e}}$ semaine de grossesse pour informer la caisse-maladie que l'embryon est bien accroché et que l'accouchement est prévu pour la 40 semaine. Ou encore de cet ami diabétologue qui s'imagine devoir, dans un avenir plus ou moins proche, remplir de longs rapports pour expliquer pourquoi un diabète juvénile insulinodépendant va nécessiter un traitement à vie.

Mais trêve de sarcasmes! Ce qui me sidère, c'est l'absence quasi totale de réactions à cette décision ahurissante. En dehors de quelques voix individuelles qui se sont exprimées dans des courriers des lecteurs, je n'ai vu aucune prise de position de la FMH ou de la Société Suisse de Psychiatrie dans les journaux de la profession. Les aurais-je manquées par mégarde, tout comme les nombreux collègues que j'ai interrogés ces dernières semaines?

Parce que, dans un domaine tout différent, on parlerait ni plus ni moins d'un autogoal. C'est effectivement le même département qui, par son chef, le conseiller fédéral Pascal Couchepin, se plaint d'une part que les troubles psychiques chroniques envahissent l'Assurance Invalidité, et qui, d'autre part, veut limiter les traitements qui permettraient de maintenir les personnes concernées au travail.

L'argument de Monsieur D. Dauwalder, chargé de communication au DFI, que la Suisse manquant cruellement de psychothérapeutes, un plus grand nombre de patients pourront y recourir si les traitements raccourcissent, est tout aussi spécieux! S'ils meurent, on aura aussi le moyen d'en traiter davantage ... à défaut de les soigner.
Qui peut imaginer qu'un médecin, psychiatre ou non, ne cherche pas par tous les moyens à soigner, éventuellement à guérir le plus rapidement possible ses patients dont on sait que la douleur morale engendre souvent plus de souffrance que le mal physique?

A diplôme égal, il faut six ans à un psychiatre pour arriver au bout de sa formation postgraduée et prétendre recevoir un titre de spécialiste $\mathrm{FMH}$... et 12 jours de cours «intensifs» à un médecin-conseil d'assurance, qui n'a probablement jamais mis les pieds dans une clinique psychiatrique ou un centre psychosocial, pour être capable de déterminer si un traitement est approprié ou non et s'il doit être payé par la caisse qui l'emploie. Quel gaspillage que les cinq ans, onze mois et 18 jours que le psychiatre consacre à apprendre à administrer ce traitement. Douze jours de cours intensifs supplémentaires permettraient de le rendre opérationnel beaucoup plus rapidement et de résorber ainsi plus efficacement nombre de cas en attente de psychothérapie.

La décision du DFI occulte complètement la notion de troubles psychiques chroniques ou récurrents. Ces derniers plus particulièrement nécessitent des contacts réguliers et fréquents avec le spécialiste pour pouvoir intervenir rapidement et efficacement au moment d'une rechute.

La définition même de la psychothérapie, telle qu'elle est mentionnée dans le document du 3 juillet 2006 (article 2, paragraphe 2) ouvre la porte à toutes les interprétations:

On entend par psychothérapie une forme de traitement des maladies psychiques et psychosomatiques qui repose essentiellement sur la communication orale, une théorie du comportement normal et pathologique et un diagnostic étiologique. Elle comprend la réflexion systématique et une relation thérapeutique suivie, se caractérise par des séances de thérapie régulières et planifiées et vise un objectif thérapeutique défini au moyen de techniques acquises dans le cadre d'une formation.

La composante psychique de nombre de maladies chroniques est bien connue et cette définition permettrait, s'il en venait subitement l'idée aux assureurs, de limiter le temps consacré par les généralistes à parler avec leurs patients qui 
ont besoin d'un soutien psychologique face à telle ou telle affection au long cours qui les limite dans la vie quotidienne ou professionnelle.

Il faut garder à l'esprit que le raccourcissement de la durée de séjour hospitalier des patients psychiatriques auquel on assiste depuis une trentaine d'années charge considérablement le secteur ambulatoire. Le but en est aussi politique puisqu'en agissant de la sorte, les cantons diminuent d'autant leur part aux subventions hospitalières qui se reporte sur les caisses-maladie, seules garantes du paiement des frais ambulatoires.

Malgré les progrès thérapeutiques de ces dernières décennies, la prise en charge de patients à peine stabilisés après un court séjour en clinique est extrêmement lourde et s'accommode mal d'une limitation à 40 séances. Il est évident que la prise en charge de ce type de pathologie tombe sous le coup de la définition ci-dessus, la communication orale étant inévitable en psychiatrie, comme dans le reste de la médecine. On le sait de triste mémoire, les assureurs arguent depuis longtemps du fait qu'il y a toujours une part de psychothérapie dans une séance chez le psychiatre pour réclamer des rapports médicaux réguliers, même chez des schizophrènes chroniques ou des bipolaires traités depuis des dizaines d'années.

Dans de telles conditions, il faut se demander s'il est logique d'entreprendre un traitement si sa continuation ne va pas de soi et dépend du bon vouloir d'un médecin-conseil dont l'expérience en psychiatrie se limite à quelques cours au lit du malade durant ses études. J'imagine le chirurgien que l'on autorise à intervenir, puis à qui on refuse la prise en charge des soins postopératoires, ou pire, de la suture. Mais je suis naïf. C'est déjà le cas pour la reconstruction mammaire en cas de cancer du sein ou la plastie cutanée après chirurgie baryatrique. Personne n'a de garantie que la caisse-maladie va prendre en charge les frais nécessaires pour que le sujet retrouve une apparence digne d'un être humain.
Finalement, parmi les nombreuses questions que cette décision du DFI soulève, il en est trois dont la réponse ne paraît pas évident. D'abord, on se demande quel but poursuivent les assureurs maladie et le département, puisque les médecins n'ont manifestement pas été consultés. Selon Monsieur Dauwalder, il ne s'agit pas de faire des économies, mais de permettre au plus grand nombre d'accéder à la psychothérapie. Le meilleur moyen serait de susciter des vocations pour la psychiatrie chez les étudiants en médecine. En traitant cette spécialité comme on le fait, de pourvoyeuse de l'AI, de travail de parlote, des médecins qui rendent leurs patients dépendants et j'en oublie, on ne va certes pas améliorer la situation. Ce n'est pas non plus en réduisant l'efficacité des traitements en les interrompant prématurément que l'on va donner l'impression aux patients qu'il peuvent trouver l'aide dont ils ont cruellement besoin auprès d'un psychiatre.

L'ordonnance du DFI ne précise pas non plus quel laps de temps entre deux prises en charge permet de remettre le compteur à zéro. Par exemple, combien de temps sans traitement faut-il compter entre deux épisodes dépressifs pour que le patient ait droit à une nouvelle tranche de 40 séances sans que la suite de la prise en charge soit mise en cause? L'OMS préconise une durée de traitement de la dépression de deux ans lors d'un deuxième épisode. C'est déjà bien au-delà de ce que l'ordonnance autorise sans formalités administratives contraignantes.

Enfin, nos patients et nous-mêmes devons nous attendre à ce que leur santé psychique soit subordonnée à des médecins-conseils dont la langue maternelle est rarement le français, et encore moins l'italien et qui vont porter un jugement sur la validité d'une prise en charge, puis de sa continuation, sans saisir pleinement les nuances des rapports qu'ils auront à peine le temps de lire tant ils seront débordés (comme c'est déjà le cas selon le reportage récent de la TSR).

Note de la rédaction: nous nous permettons de rappeler à notre lectorat que dans l'édition du BMS $n^{\circ}$ 29/30 du 26 juillet 2006, un éditorial a paru de Hans Kurt, président de la FMPP/SSPP, concernant les modifications de l'Ordonnance sur les prestations de l'assurance des soins (OPAS) thématisées dans le texte de B. Scherler. L'introduction à cet éditorial était de la plume de René Raggenbass, membre du Comité central de la FMH. 